\title{
Implications of Ultrasound Mismatch: A Novel Case Report of Discrepancy between Bedside Versus Formal Biliary Ultrasound
}

Keywords: Ultrasound; Choledocholithiasis; Gallstone; Common bile duct; Emergency department; Bedside ultrasound; Point of care

\begin{abstract}
Ultrasound (US) is the gold standard imaging modality utilized to diagnose biliary disease. Point of care (POC) US in the emergency department (ED) can and has played an integral role in assessing patients for biliary disease to accelerate care. We report a case where bedside US in the ED was used as an important aspect of the patient's initial work up. Our case involves a 29 year-old female who presented to the emergency department with skin jaundice and sclera icterus. The patient had a history of liver transplant for hepatoblastoma at age 22 months, and was advised to come to the ED when her primary care physician noticed elevated liver enzymes on a routine lab check. When presenting to the emergency department, the patient denied any abdominal pain or nausea, but was experiencing dark colored urine and tan colored stools. Due to the suspicious nature of the patient's symptoms and history of present illness, a POC US in the ED was done which demonstrated a dilated common bile duct (CBD) secondary to visible stone, however the follow up complete abdominal ultrasound in the radiology suite subsequently was negative for pathology. Ultimately the patient received a magnetic resonance cholangiopancreatography (MRCP), demonstrated a gallstone in the common bile duct and reaffirmed the findings on POC US. The gallstone was removed and the patient received a revision of her hepaticojunostomy. This case demonstrates pathology recognized by the POC US performed in the ED, that was absent on radiology US, suggesting a transient and dynamic change in the gallstone's positioning within the duct.
\end{abstract}

\section{Introduction}

The utilization of ultrasound (US) for evaluating biliary disease has been well established [1,2]. Biliary disease includes the presence of gallstone(s) or a gallstone within the common bile duct (CBD). Moreover, the benefits of POC US in the emergency department (ED) to assess for biliary disease have been illustrated in previous literature [3-7]. The true prevalence of gallbladder disease is unknown, however it is estimated that over twenty million Americans suffer from some variation of gallbladder disease [8]. Many of these patients will be seen and assessed in the ED with associated abdominal pain. We present a case of choledocholithiasis where CBD dilation was demonstrated on the initial ED POC US, but was negative with the subsequent radiology US. The discovery of an obstructing gallstone dilating the $\mathrm{CBD}$ was later confirmed via magnetic resonance cholangiopancreatography (MRCP), and removed surgically during revision of hepaticojunostomy. POC US in the ED played an integral role in the work up of this clinical scenario, ultimately correlating with MRCP findings, and leading to the appropriate treatment.

\section{Case Report}

The patient is a 29 year-old female who presents to the ED with

\section{Journal of}

Emergency Medicine \& Critical Care

\author{
Michael J. Burla ${ }^{1,2 *}$, David A. Berger ${ }^{1,2}$, Abigail \\ Brackney ${ }^{1,2}$ and Amit Bahl ${ }^{1,2}$
}

${ }^{1}$ Department of Emergency Medicine, William Beaumont Hospital, Royal Oak, Michigan, USA

2Oakland University, William Beaumont School of Medicine, Rochester, Michigan, USA

\section{Address for Correspondence}

Michael Burla D.O, Department of Emergency Medicine, William Beaumont Hospital, Oakland University, 3601 W 13 Mile Rd, Royal Oak, Michigan, USA 48073, Tel: 248-898-9111; E-mail: Michael. Burla@beaumont.edu
Submission: 22 August, 2015
Accepted: 18 September, 2015
Published: 22 September, 2015
Copyright: $\odot 2015$ Burla MJ, et al. This is an open access article distributed under the Creative Commons Attribution License, which permits unrestricted use, distribution, and reproduction in any medium, provided the original work is properly cited.
Reviewed \& Approved by: Dr. Adeyinka Adedipe, Assistant Professor, Division of Emergency Medicine, University of Washington, USA

mild jaundice and scleral icterus. She was sent in by her primary care physician due to abnormal liver function enzymes on routine lab work one day ago. The patient had a liver transplant for hepatoblastoma at age 22 months old, but had an otherwise unremarkable past medical history. The patient's jaundice and scleral icterus has been gradually developing, and she states that she has also been experiencing dark colored urine and tan colored stools. The patient was not experiencing abdominal pain, and denied any other symptoms, such as chest pain, difficulty with breathing, coughing, fever, nausea, or vomiting.

On physical examination the vital signs were: blood pressure of $125 / 78 \mathrm{mmHg}$, heart rate of 99 beats per minute, respiratory rate of 20 breaths per minute, temperature of $36.2^{\circ} \mathrm{C}$, and a pulse oximetry of $99 \%$ on room air. The patient appeared well in the ED, oriented to person, place, and time. The patient's skin and eye exam were positive for jaundice and scleral itcerus, respectively. Examination of the abdomen demonstrated a non-tender and well healed transverse abdominal incision, without evidence or right upper quadrant tenderness and a negative murphy's sign. The rest of the patient's physical exam was unremarkable. Given the patient's presentation and history of recent abnormal lab work, blood work was done which demonstrated an alkaline phosphatase level of 585, aspartate aminotransferase of 280, alanine aminotransferase of 162, and a direct bilirubin of 4.2. Due to the abnormal lab work and physical findings, possible biliary disease with an obstruction was considered, and a POC US was performed at bedside in the ED. The findings of the POC US demonstrated a dilated CBD at $1.93 \mathrm{~cm}$ and gallstone within the CBD, illustrated in Figure 1 and Figure 2 respectively. The patient was admitted to the hospital, and a complete abdominal ultrasound was then ordered at the request of the admitting service. However, the radiology US demonstrated an unremarkable CBD, with a measurement of $5 \mathrm{~mm}$ illustrated in Figure 3. Additionally no gallstone was identified in the CBD or gallbladder. The radiology US 
Citation: Burla MJ, Berger DA, Brackney A, Bahl A. Implications of Ultrasound Mismatch: A Novel Case Report of Discrepancy between Bedside Versus Formal Biliary Ultrasound. J Emerg Med Critical Care. 2015;1(1): 3.

was performed within one hour of the POC US, suggesting a dynamic change within the CBD.

During the patient's hospital stay, consultations were made to gastroenterology, hepatology, nephrology, and transplant surgery. Additional work up was negative for hepatitis or transplant rejection. A magnetic resonance cholangiopancreatography (MRCP) was performed and demonstrated a large stone within the proximal

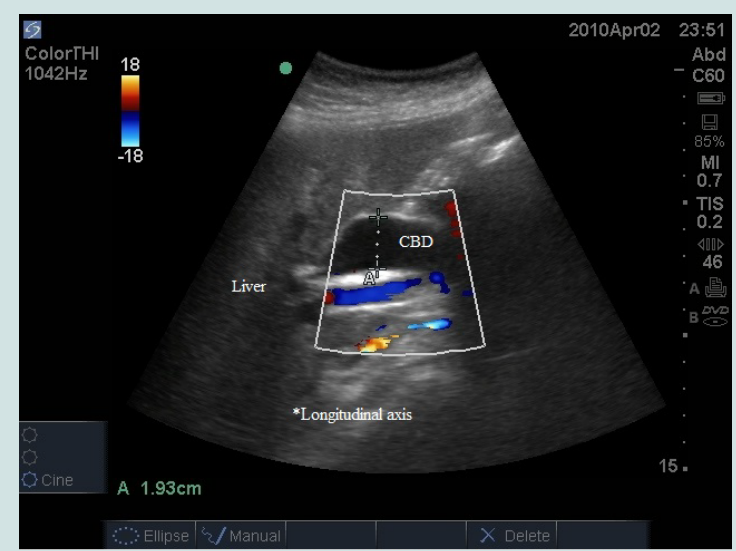

Figure 1: $P O C$ US performed in $E D$, illustrating dilated $C B D$ with measurement $A$, at $1.93 \mathrm{~cm}$.

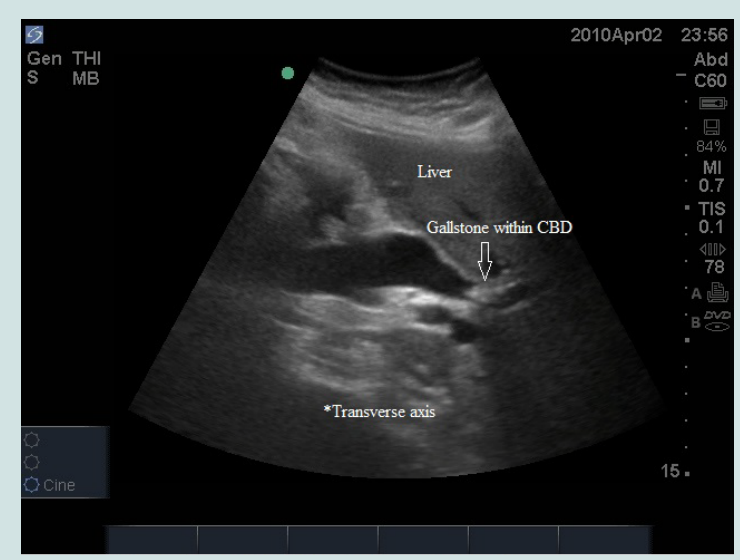

Figure 2: POC US performed in ED, illustrating gallstone within the CBD.

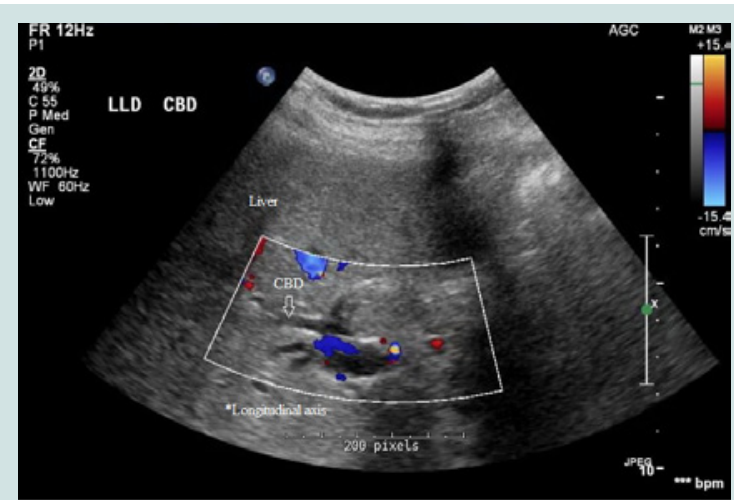

Figure 3: Radiology US performed in the formal fashion, illustrating a non dilated $\mathrm{CBD}$ without evidence of gallstone.
$\mathrm{CBD}$, measuring $3 \times 1.8 \mathrm{~cm}$. The gallstone was removed after CBD exploration and revision of her hepaticojunostomy. The patient's surgery was unremarkable and several days after the procedure her liver function enzymes reduced to within normal limits. The patient was discharged home after receiving proper treatment of her condition, and a resolution of her symptoms.

\section{Discussion}

This case illustrates a unique situation, where a dynamic change in $\mathrm{CBD}$ was observed within on one hour of presentation. Early POC US in the ED demonstrated an obstructing gallstone in the CBD, with the follow-up radiology US demonstrating a CBD diameter within normal limits. After admission the correct diagnosis of choledocholilthasis was confirmed with MRCP, the patient received definitive treatment of her condition. Previous literature has described cases of choledocholilthasis in unique settings, but almost none have demonstrated a case where the diagnosis was recognized on bedside US in the ED and not on follow-up radiology US. One case described by Jafari et al. demonstrated a fluctuating CBD diameter over a 72 hour period, with an initial dilated CBD on bedside US of $17 \mathrm{~mm}$ [9]. However, this CBD fluctuation was evident on multiple imagining modalities over the patient's hospital stay, with the first radiology US being performed five hours after POC US. In addition, the patient had correlating symptoms of nausea, vomiting, and abdominal pain with CBD dilation, with a resolution of those symptoms when US demonstrated a CBD diameter within normal limits [9]. Our case differs from this case due to the fact that our patient did not experience any fluctuating symptoms of nausea, vomiting, and abdominal pain. Moreover, our patient was experiencing jaundice and sclera icterus without abdominal pain, which is atypical for an obstructing gallstone in the $\mathrm{CBD}$. The transient changes in $\mathrm{CBD}$ diameter of our case within an hour suggest that dynamics of gallstone mobilization within a CBD can be very acute in nature. To our knowledge, there is no other documentation of a case similar to our case in previous literature.

Although our case is distinguishable from previous publications, it reinforces the benefit of POC US in the ED. Given the dynamic changes of the $\mathrm{CBD}$ in the presence of biliary disease, proper use of bedside US in the ED can provide early recognition of pathology and guide treatment. The implications for these dynamics suggest that multiple scans may bare different results, which is a factor clinicians should consider. Previous research has described sufficient abilities of non-fellowship trained physicians in recognizing biliary disease with bedside US $[6,10]$, including Turner et al. describing that curriculum based simulated US programs can adequately provide emergency medicine physicians with US skills within a short period of time [10]. Additionally, Summers et al. demonstrated POC US in the ED had similar detection of cholecystitis comparted to radiology US [6]. With this understanding, the utility of POC US has been universally accepted as a means of obtaining additional information, especially when admission is warranted. Given the proper clinical context, emergency physicians should consider performing a bedside US study when biliary disease is suspected.

\section{References}

1. Panella J, Rochester D, Williams R, Bernstein J (1980) Ultrasonic diagnosis of intrahepatic biliary stones. JAMA 243: 2155-2156. 
Citation: Burla MJ, Berger DA, Brackney A, Bahl A. Implications of Ultrasound Mismatch: A Novel Case Report of Discrepancy between Bedside Versus Formal Biliary Ultrasound. J Emerg Med Critical Care. 2015;1(1): 3.

2. Weinstein DP, Weinstein BJ, Brodmerkel GJ (1979) Ultrasonography of biliary tract dilatation without jaundice. AJR Am J Roentgenol 132: 729-734.

3. Ross M, Brown M, McLaughlin K, Atkinson P, Thompson J, et al. (2011) Emergency physician-performed ultrasound to diagnose cholelithiasis: a systematic review. Acad Emerg Med 18: 227-235.

4. Kogan I, Barzilai N, Hay E, Fruman A (1995) Ultrasound evaluation of acute abdominal pain in the emergency department. Harefuah 128: 484-487, 527.

5. Jehle D, Davis E, Evans T, Harchelroad F, Martin M, et al. (1989) Emergency department sonography by emergency physicians. Am J Emerg Med 7: 605611.

6. Summers SM, Scruggs W, Menchine MD, Lahham S, Anderson C, et al., (2010) A prospective evaluation of emergency department bedside ultrasonography for the detection of acute cholecystitis. Ann Emerg Med 56 114-122.
7. Miller AH, Pepe PE, Brockman CR, Delaney KA (2006) ED Ultrasound in hepatobiliary disease. J Emerg Med 30: 69-74.

8. Everhart JE, Khare M, Hill M, Maurer KR (1999) Prevalence and ethnic differences in gallbladder disease in the United States. Gastroenterology 117 632-639.

9. Jafari D, Cheng AB, Dean AJ (2013) Dynamic changes of common bile duct diameter during an episode of biliary colic, documented by ultrasonography. Ann Emerg Med 62: 176-179.

10. Turner EE, Fox JC, Rosen M, Allen A, Rosen S, et al. (2015) Implementation and assessment of a curriculum for bedside ultrasound training. J Ultrasound Med 34: 823-828.

\section{Acknowledgements}

We would like to thank the William Beaumont Hospital (Royal Oak, MI) emergency department, as well as all the nurses and physicians that played a role in this patient's care. 